\title{
Medical status of patients presenting for treatment at an Australian dental institute: a cross-sectional study
}

\author{
Agnieszka M. Frydrych ${ }^{1 *} \mathbb{D}$, Richard Parsons ${ }^{2}$ and Omar Kujan ${ }^{1}$
}

\begin{abstract}
Background: Individuals seeking dental treatment often present with medical conditions which may affect dental treatment provision. The purpose of this study was to determine the prevalence of medical conditions and medication use among individuals attending dental clinics at a Western Australian tertiary institution.

Methods: This observational study examined the general demographics, medical and social histories of 873 subjects presenting for a general dental examination at a Western Australian Tertiary Institution between March 2018 and February 2019. Individual recruited were those presenting to the clinics either as new patients to the Centre or returning patients after an extended period of absence.

Results: Presence of a medical condition was reported by $86 \%$ of the participants, with males more commonly affected ( $p=0.0448$ ). Medication use was noted by $80 \%$ of the participants. Cardiovascular disease (37.9\%), allergy (32.3\%), mental health disorders (29.4\%), musculoskeletal (23.0\%), endocrine (22.4\%) and respiratory disease (17.8\%) were the most common conditions identified. Women were significantly more likely to report allergy $(p=0.0002)$ or a mental health condition $(p=0.0368)$.

Conclusion: Medical comorbidities are common among individuals presenting for student dental treatment in Western Australia, highlighting the importance of knowledge and teaching of internal medicine and its application to the dental setting.
\end{abstract}

Keywords: Systemic diseases, Dentistry, Dental institute, Western Australia, Cross-sectional

\section{Background}

Individuals seeking dental treatment often present with underlying medical conditions, which may or may not be managed with a variety of medications [1-16]. These comorbidities impact on the delivery of dental treatment and the importance of understanding the health status of the dental patient is well recognised [17-19]. The safety and effectiveness of dental treatment provision rests on the degree to which an individual's medical status

\footnotetext{
*Correspondence: agnieszka.frydrych@uwa.edu.au

1 UWA Dental School, The University of Western Australia, 17 Monash

Avenue, Nedlands, WA 6009, Australia
}

Full list of author information is available at the end of the article is considered in dental treatment planning. This consideration in turn is highly dependent on the clinician's knowledge of internal medicine and its application to the dental setting. Shortcomings of the dental curricular with respect to the preparation of students to deal with patients with underlying medical conditions have been demonstrated in a number of studies [20,21].

Although grounding in general medicine is essential for the dental students in the modern era; understanding the most commonly encountered medical conditions in the dental setting, has the potential to shift the focus of internal medicine teaching. This may motivate students to learn internal medicine through interest and appreciation of relevance of subjects discussed. Focusing teaching 
around student experience may also help the students to better contextualize their theoretical knowledge [22].

A number of studies have been undertaken with the aim of ascertaining the types of medical conditions prevalent among dental patients in general $[1,8,10]$ and specialist practice $[9,10,12,14,15]$ as well as in dental school patient populations [2, 3, 5-7, 9-14, 16]. The prevalence of medical problems among dental patients has been shown to vary greatly, ranging from 1 to $83 \%$, depending on the population studied [1-3, 6-10, 12-16]. Elderly patients and those attending public dental facilities have been shown to demonstrate increased prevalence of systemic disease compared to their younger counterparts or those seeking treatment in the private sector $[10,12,13]$. While a broad range of comorbidities has been reported, cardiovascular disease [5-10, $12-14,16]$, respiratory conditions $[2,3,5,7,8,10-12$, $14,16]$, endocrine disorders $[1-3,6,7,10-14,16]$, bleeding pathosis $[1,5,11]$, arthritis $[2,6-8,11-13,16]$, and allergies $[1,2,6,7,9-11,13,16]$ constitute the most commonly reported medical problems encountered in the dental settings. Despite fewer studies reporting medication use, the most commonly prescribed medications parallel the most frequently encountered medical problems, with medications used in the management of cardiovascular disease the most commonly reported $[6,7$, 13, 15].

At present there is a general paucity of Australian data $[8,10]$ and a lack of data concerning the medical status of patients seen by the dental students in Western Australia. In view of this, the aims of this study were to 1 . determine the prevalence of medical conditions and medication use among dental patients attending the general dental clinics for a routine dental examination at the Oral Health Centre of Western Australia (OHCWA), University of Western Australia and 2. present associations between patient demographics and social habits and subject's medical history and medication use. The prevalence estimates are documented and compared between males and females because differences in some of these are known to exist between genders $[2,5,7,13,14]$. It is anticipated that results of this study will shed light on the types of comorbidities of dental patients encountered by the dental students during their clinical studies, thus stimulating discussion around internal medicine content delivery, with the view to better prepare the graduates to deal with the medically compromised patients.

\section{Methods}

This was an observation, cross-sectional study based on examination of existing patient records approved by the Human Research Ethics Committee of the University of Western Australia (RA/4/20/4435) and has been reported in accordance with the Strengthening the Reporting of Observational Studies in Epidemiology (STROBE) statement [23].

Study participants were recruited from a pool of patients presenting to the general dental clinics at the Oral Health Centre of Western Australia (OHCWA), University of Western Australia between March 2018 and February 2019. Individual recruited were those presenting to the clinics either as new patients to the Centre or returning patients after a period of absence of at least 12 months. From the administrative and assessment perspective, patients representing to the Centre for treatment after a period of absence are treated the same as new patients. The institution represents the only centralised public tertiary referral centre in Western Australia, where eligible patients are treated on behalf of the State Government. Attendance at the OHCWA is governed by strict eligibility criteria based on an individual's socioeconomic status, which are checked each time an individual attends the Centre. For an individual to continue to receive treatment at the Centre, they must continue to meet the eligibility criteria. All new patients and those representing after a period of absence seeking to obtain treatment at the Centre undergo a dental examination and a comprehensive assessment of their medical and social history, which is recorded in the Centre's Titanium Oral Health Management (TOHM) system. We focused on new patients presenting to the Centre as this allowed us to collect the subjects' most comprehensive and up to date social and medical history data and minimized the problem of incomplete or inaccurate records. Throughout the study period, we regularly audited the clinicians to ensure that the patient history was accurately and fully recorded. All new patients presenting to the Centre (and those returning after a period of absence) during the study period were enrolled in the study with the choice to opt-out from participation. Participation was voluntary, and participants were at liberty to withdraw from the study at any time, with no disadvantage to their ongoing treatment.

The study subjects were individuals aged 25 years or older attending the OHCWA general dental clinics for a routine dental examination. All individuals with unknown medical history or opting out from participation in the study were excluded. The recorded variables for each subject were derived from the data recorded in TOHM and included: age, gender, residential post code, IRSAD (socioeconomic status score, based on residential postcode), medical history (including drug history) as well as smoking and alcohol use. 


\section{Statistical analysis}

Following record de-identification, all data were manually entered into a Microsoft Excel spreadsheet (Redmond, WA, USA) and verified independently to prevent data transfer error. The data were then exported into SAS version 9.4 (SAS Institute Inc, Cary, NC, USA 2008) for analysis. Simple descriptive analyses (frequencies and percentages) were used to summarise the demographic and medical profile of the study participants. Chi-square statistics were used to compare these profile variables between male and female participants (univariate analyses). In comparing the two genders, odds ratios for females compared to males were calculated for each variable, along with its $95 \%$ confidence interval. The mean IRSAD was compared between genders for medical history and medication use, using the Student's t-test. Statistical significance was set at $p<0.05$ in all tests.

\section{Results}

One thousand and four subjects were approached for inclusion in the study. A total of 131 were excluded from the study, either because they chose not to participate or their medical history was incomplete, so that a total of 873 subjects were included in the final analysis. The general characteristics of all participants, and divided according to gender, are presented in Table 1.Male subjects were more likely to use tobacco $(p<0.0001)$ and consume alcohol $(p=0.0002)$. Eighty six percent of study participants reported having a medical condition, with males more likely to be affected ( $p=0.0448)$.

Table 2 illustrates the prevalence of disease and medication usage among the study participants. Cardiovascular disease (37.9\%), allergy (32.3\%), mental health disorders (29.4\%) musculoskeletal (23.0\%) endocrine (22.4\%) and respiratory disease $(17.8 \%)$ constituted the most common conditions reported. Women were significantly more likely to report allergy $(p=0.0002)$ or a mental health condition $(p=0.0368)$. No gender differences were observed amongst the remaining commonly reported pathosis.

Medication use was reported by $80 \%$ of the study participants with the pattern of medication usage paralleling the medical conditions reported. Antidepressants were the medications most commonly used by the individuals presenting for treatment with $22.6 \%$ of all subjects reporting the use of such medications. Women were twice as likely to report taking antidepressant medication compared to their male counterparts $(p=0.0001$; odds ratio [95\% CI] 1.93 [1.38-2.69]). Analgesics (17.2\%), anticoagulants (17.2\%) and drugs for dyslipidaemia (20.7\%) were also commonly used in the population studied.
Table 1 General characteristics of the participants

\begin{tabular}{|c|c|c|c|c|}
\hline Variable & $\begin{array}{l}\text { Total } \\
\mathrm{N}=873\end{array}$ & $\begin{array}{l}\text { Male } \\
\mathrm{N}=394(\%)\end{array}$ & $\begin{array}{l}\text { Female } \\
\mathrm{N}=479 \text { (\%) }\end{array}$ & $\begin{array}{l}p \text { value } \\
\text { (Chi-square } \\
\text { test) }\end{array}$ \\
\hline Age group (years) & & & & 0.4519 \\
\hline $25-34$ & 105 & $40(10.2)$ & 65 (13.6) & \\
\hline $35-44$ & 131 & $54(13.7)$ & $77(16.1)$ & \\
\hline $45-54$ & 137 & $61(15.5)$ & $76(15.9)$ & \\
\hline $55-64$ & 147 & $66(16.8)$ & $81(16.9)$ & \\
\hline $65-74$ & 228 & $112(28.4)$ & $116(24.2)$ & \\
\hline $75-84$ & 103 & $52(13.2)$ & $51(10.7)$ & \\
\hline $85+$ & 22 & $9(2.3)$ & $13(2.7)$ & \\
\hline Tobacco use & & & & $<0.0001$ \\
\hline Never & 481 & $168(42.6)$ & $313(65.5)$ & \\
\hline Current & 201 & $109(27.7)$ & $92(19.3)$ & \\
\hline Ex-user & 190 & $117(29.7)$ & $73(15.3)$ & \\
\hline \multicolumn{3}{|c|}{ Tobacco (current/ex-user) } & & $0.5061^{*}$ \\
\hline Self-rolled & 19 & $10(4.4)$ & $9(5.5)$ & \\
\hline Cigarette & 370 & $214(94.7)$ & $156(94.6)$ & \\
\hline $\begin{array}{l}\text { Chew/vape/ } \\
\text { other }\end{array}$ & 2 & $2(0.9)$ & $0(0.0)$ & \\
\hline Alcohol use & & & & 0.0002 \\
\hline Never & 457 & $177(44.9)$ & $280(58.6)$ & \\
\hline Current & 393 & $203(51.5)$ & $190(39.8)$ & \\
\hline Ex-user & 22 & $14(3.6)$ & $8(1.7)$ & \\
\hline Alcohol by type & & & & 0.0001 \\
\hline Spirits & 25 & $14(9.3)$ & $11(9.1)$ & \\
\hline Wine & 133 & $56(37.1)$ & 77 (63.6) & \\
\hline Beer & 59 & $45(29.8)$ & $14(11.6)$ & \\
\hline Cider & 6 & $3(2.0)$ & $3(2.5)$ & \\
\hline Mixed & 49 & $33(21.9)$ & $16(13.2)$ & \\
\hline
\end{tabular}

The $p$ value compares each variable between male and female respondents *Fisher's Exact test

The effect of age group, gender, smoking and alcohol use on reporting a positive medical history and medication use is shown in Table 3. Young males (age group 25-34) were significantly more likely to report a positive medical history compared to females in the same age group $(p=0.0069)$. Among the current tobacco users, men were significantly more likely to present with a medical condition compared to their female counterparts $(p=0.0340)$. Among the nondrinkers, more men than women described having a medical condition $(p=0.0383)$. Compared to males (Table 4$)$, women from lower socioeconomic background were significantly less likely to use medication $(p=0.0298)$.

\section{Discussion}

Individuals seeking dental treatment often present with medical comorbidities, which affect the safety and effectiveness of dental treatment provision. Clinician's 
Table 2 The prevalence of disease and medication usage among the cases

\begin{tabular}{|c|c|c|c|c|c|}
\hline Variable & $\begin{array}{l}\text { Total } \\
\mathrm{N}=873(\%)\end{array}$ & $\begin{array}{l}\text { Males } \\
\mathrm{N}=394 \text { (\%) }\end{array}$ & $\begin{array}{l}\text { Females } \\
\mathrm{N}=479(\%)\end{array}$ & $\begin{array}{l}\text { Odds ratio (Female relative } \\
\text { to Male) }(95 \% \mathrm{Cl})\end{array}$ & $\begin{array}{l}p \text { value (Chi- } \\
\text { square test) }\end{array}$ \\
\hline \multicolumn{6}{|l|}{ Disease characteristics } \\
\hline Presence of disease & $753(86.3)$ & $350(88.8)$ & $403(84.1)$ & $0.67(0.45-0.99)$ & 0.0448 \\
\hline Allergy & $282(32.3)$ & $102(25.9)$ & $180(37.6)$ & $1.72(1.29-2.31)$ & 0.0002 \\
\hline Auditory system disorder & $11(1.3)$ & $5(1.3)$ & $6(1.3)$ & $0.99(0.30-3.26)$ & $1.0^{*}$ \\
\hline Blood disorder & $23(2.6)$ & $6(1.5)$ & $17(3.6)$ & $2.38(0.93-6.09)$ & 0.0629 \\
\hline Cancer & $100(11.5)$ & $48(12.2)$ & $52(10.9)$ & $0.88(0.58-1.33)$ & 0.5402 \\
\hline Cardiovascular disease & $331(37.9)$ & $153(38.8)$ & $178(37.2)$ & $0.93(0.71-1.23)$ & 0.6124 \\
\hline Gastrointestinal system disorder & $118(13.5)$ & $51(12.9)$ & $67(14.0)$ & $1.09(0.74-1.62)$ & 0.6537 \\
\hline Endocrine system disorder & $196(22.4)$ & $81(20.6)$ & $115(24.0)$ & $1.22(0.88-1.68)$ & 0.2241 \\
\hline Diabetes & $136(15.6)$ & $63(16.0)$ & $73(15.2)$ & $0.94(0.65-1.36)$ & 0.7611 \\
\hline Dyslipidaemia & $38(4.4)$ & $15(3.8)$ & $23(4.8)$ & $1.27(0.66-2.48)$ & 0.4736 \\
\hline Other endocrine & $49(5.6)$ & $10(2.5)$ & $39(8.1)$ & $3.40(1.68-6.91)$ & 0.0003 \\
\hline Immune system disorder & $38(4.4)$ & $19(4.8)$ & $19(4.0)$ & $0.82(0.43-1.56)$ & 0.5377 \\
\hline Zoonotic diseases & $15(1.7)$ & $13(3.3)$ & $2(0.4)$ & $0.12(0.03-0.55)$ & 0.0011 \\
\hline Liver disease & $15(1.7)$ & $7(1.8)$ & $8(1.7)$ & $0.94(0.34-2.61)$ & 0.9041 \\
\hline Mental health disorder & $257(29.4)$ & $102(25.9)$ & $155(32.4)$ & $1.37(1.02-1.84)$ & 0.0368 \\
\hline Musculoskeletal system disorder & $201(23.0)$ & $92(23.4)$ & $109(22.8)$ & $0.97(0.70-1.33)$ & 0.8355 \\
\hline Nervous system & $108(12.4)$ & $51(12.9)$ & $57(11.9)$ & $0.91(0.61-1.36)$ & 0.6409 \\
\hline Renal system & $29(3.3)$ & $13(3.3)$ & $16(3.3)$ & $1.01(0.48-2.13)$ & 0.9733 \\
\hline Reproductive system & $35(4.0)$ & $9(2.3)$ & $26(5.4)$ & $2.46(1.14-5.30)$ & 0.0185 \\
\hline Respiratory system disorder & $155(17.8)$ & $68(17.3)$ & $87(18.2)$ & $1.06(0.75-1.51)$ & 0.7280 \\
\hline Asthma & $5(10.9)$ & $39(9.9)$ & $56(11.7)$ & $1.21(0.78-1.86)$ & 0.3974 \\
\hline COPD & $32(3.4)$ & $18(4.6)$ & $14(2.9)$ & $0.63(0.31-1.28)$ & 0.1978 \\
\hline Other & $41(4.7)$ & $17(4.3)$ & $24(5.0)$ & $1.17(0.62-2.21)$ & 0.6287 \\
\hline Skin conditions & $26(3.0)$ & $14(3.6)$ & $12(2.5)$ & $0.70(0.32-1.53)$ & 0.3646 \\
\hline Viral infections & $48(5.5)$ & $26(6.6)$ & $22(4.6)$ & $0.68(0.38-1.22)$ & 0.1957 \\
\hline Visual system disorder & $35(4.0)$ & $16(4.1)$ & $19(4.0)$ & $0.98(0.50-1.92)$ & 0.9436 \\
\hline Other disease & $5(0.6)$ & $1(0.3)$ & $4(0.8)$ & $3.31(0.37-29.73)$ & $0.3854^{*}$ \\
\hline \multicolumn{6}{|l|}{ Medication usage } \\
\hline Taking Medication & $699(80.0 \%)$ & $317(80.5)$ & $382(79.9)$ & $0.97(0.69-1.35)$ & 0.8421 \\
\hline Ace inhibitor & $86(9.9 \%)$ & $51(12.9)$ & $35(7.31)$ & $0.53(0.34-0.83)$ & 0.0054 \\
\hline Alpha Blockers & $5(0.6)$ & $4(1.0)$ & $1(0.2)$ & $0.20(0.02-1.83)$ & $0.1811^{*}$ \\
\hline Analgesics & $150(17.2)$ & $59(15.0)$ & $91(19.0)$ & $1.33(0.93-1.91)$ & 0.1168 \\
\hline Opioid & $69(7.9)$ & $36(9.1)$ & $33(6.9)$ & $0.74(0.45-1.20)$ & 0.2206 \\
\hline Non-opioid & $106(12.1)$ & $34(8.6)$ & $72(15.0)$ & $1.87(1.22-2.88)$ & 0.0040 \\
\hline Antiarrhythmic & $7(0.8)$ & $2(0.5)$ & $5(1.0)$ & $2.07(0.40-10.71)$ & $0.4668^{*}$ \\
\hline Anticoagulants & $150(17.2)$ & $84(21.3)$ & $66(13.8)$ & $0.59(0.41-0.84)$ & 0.0033 \\
\hline Anticonvulsants and antiepileptics & $73(8.4)$ & $27(6.9)$ & $46(9.6)$ & $1.44(0.88-2.37)$ & 0.1440 \\
\hline Antidepressants & $197(22.6)$ & $65(16.5)$ & $132(27.6)$ & $1.93(1.38-2.69)$ & 0.0001 \\
\hline SNRI & $39(4.5)$ & $15(3.8)$ & $24(5.0)$ & $1.33(0.69-2.58)$ & 0.3918 \\
\hline SSRI & $79(9.0)$ & $23(5.8)$ & $56(11.7)$ & $2.14(1.29-3.54)$ & 0.0027 \\
\hline Tricyclic & $25(2.9)$ & $7(1.8)$ & $18(3.8)$ & $2.16(0.89-5.22)$ & 0.0807 \\
\hline Combination & $22(2.5)$ & $10(2.5)$ & $12(2.5)$ & $0.99(0.42-2.31)$ & 0.9754 \\
\hline Other & $32(3.7)$ & $10(2.5)$ & $22(4.6)$ & $1.85(0.86-3.95)$ & 0.1079 \\
\hline Antihistamine & $19(2.2)$ & $9(2.3)$ & $10(2.1)$ & $0.91(0.37-2.27)$ & 0.8466 \\
\hline Antihypertensive NOS & $38(4.3)$ & $24(6.1)$ & $14(2.9)$ & $0.46(0.24-0.91)$ & 0.0224 \\
\hline Anti-inflammatories & $74(8.5)$ & $25(6.4)$ & 49 (10.2) & $1.68(1.02-2.78)$ & 0.0403 \\
\hline Corticosteroid & $26(3.0)$ & $10(2.5)$ & $16(3.3)$ & $1.33(0.60-2.96)$ & 0.4878 \\
\hline NSAID & $51(5.8)$ & $16(4.1)$ & $35(7.3)$ & $1.86(1.01-3.42)$ & 0.0419 \\
\hline
\end{tabular}


Table 2 (continued)

\begin{tabular}{|c|c|c|c|c|c|}
\hline Variable & $\begin{array}{l}\text { Total } \\
\mathrm{N}=873(\%)\end{array}$ & $\begin{array}{l}\text { Males } \\
\mathrm{N}=394(\%)\end{array}$ & $\begin{array}{l}\text { Females } \\
\mathrm{N}=479 \text { (\%) }\end{array}$ & $\begin{array}{l}\text { Odds ratio (Female relative } \\
\text { to Male) }(95 \% \mathrm{Cl})\end{array}$ & $\begin{array}{l}p \text { value (Chi- } \\
\text { square test) }\end{array}$ \\
\hline Antiemetics & $11(1.3)$ & $2(0.5)$ & $9(1.9)$ & $3.76(0.81-17.5)$ & $0.1238^{*}$ \\
\hline Antimicrobial & $35(4.0)$ & $15(3.8)$ & $20(4.2)$ & $1.10(0.56-2.18)$ & 0.7825 \\
\hline Antipsychotic & $60(6.9)$ & $27(6.9)$ & $33(6.9)$ & $1.01(0.59-1.70)$ & 0.9830 \\
\hline Beta blockers & $91(10.4)$ & $45(11.4)$ & $46(9.6)$ & $0.82(0.53-1.27)$ & 0.3817 \\
\hline Bisphosphonates & $9(1.0)$ & $3(0.8)$ & $6(1.3)$ & $1.65(0.41-6.65)$ & $0.5242^{*}$ \\
\hline Calcium channel blockers & $55(6.3)$ & $31(7.9)$ & $24(5.0)$ & $0.62(0.36-1.07)$ & 0.0838 \\
\hline Chemotherapeutics & $7(0.8)$ & $4(1.0)$ & $3(0.6)$ & $0.61(0.14-2.76)$ & $0.7072^{*}$ \\
\hline Diuretics & $34(3.89)$ & $18(4.6)$ & $16(3.3)$ & $0.72(0.36-1.43)$ & 0.3506 \\
\hline Anxiety and sleep disorders & $66(7.6)$ & $30(7.6)$ & $36(7.5)$ & $0.99(0.60-1.63)$ & 0.9563 \\
\hline Drugs for Diabetes & $105(12.0)$ & $55(14.0)$ & $50(10.4)$ & $0.72(0.48-1.08)$ & 0.1115 \\
\hline Drugs for dyslipidaemia & $181(20.7)$ & $98(24.9)$ & $83(17.3)$ & $0.63(0.46-0.88)$ & 0.0062 \\
\hline Statin & $156(17.9)$ & 85 (21.6) & $71(14.8)$ & $0.63(0.45-0.90)$ & 0.0096 \\
\hline Fibrate & $15(1.7)$ & $8(2.0)$ & $7(1.5)$ & $0.72(0.26-1.99)$ & 0.5197 \\
\hline Other & $18(2.1)$ & $8(2.0)$ & $10(2.1)$ & $1.03(0.40-2.63)$ & 0.9528 \\
\hline Gastrointestinal NOS & $9(1.0)$ & $5(1.3)$ & $4(0.8)$ & $0.66(0.17-2.46)$ & 0.5276 \\
\hline Genitourinary & $23(2.6)$ & $18(4.6)$ & $5(1.0)$ & $0.22(0.08-0.60)$ & 0.0012 \\
\hline $\mathrm{H} 2$ antagonists & $9(1.0)$ & $4(1.0)$ & $5(1.0)$ & $1.03(0.27-3.86)$ & $1.0^{*}$ \\
\hline Hormones & $111(12.7)$ & $23(5.8)$ & $88(18.4)$ & $3.63(2.25-5.87)$ & $<0.0001$ \\
\hline Immunosupressants & $36(4.1)$ & $17(4.3)$ & $19(4.0)$ & $0.92(0.47-1.79)$ & 0.7969 \\
\hline Inhaled & $106(12.1)$ & $42(10.7)$ & $64(13.4)$ & $1.29(0.85-1.96)$ & 0.2240 \\
\hline Laxatives & $10(1.1)$ & $6(1.5)$ & $4(0.8)$ & $0.54(0.15-1.94)$ & $0.3600^{*}$ \\
\hline Muscle relaxants & $5(0.6)$ & $3(0.8)$ & $2(0.4)$ & $0.55(0.09-3.29)$ & $0.6626^{*}$ \\
\hline Nitrates and anti-anginal & $18(2.1)$ & $9(2.3)$ & $9(1.9)$ & $0.82(0.32-2.08)$ & 0.6749 \\
\hline Other drugs affecting bone & $21(2.4)$ & $4(1.0)$ & $17(3.6)$ & $3.59(1.20-10.75)$ & 0.0150 \\
\hline
\end{tabular}

*Fisher's Exact test

knowledge of internal medicine and its application to the dental setting is therefore paramount [6]. This begins with and is facilitated by appropriate dental curriculum content delivery [22]. The immense and ever-increasing medical knowledge poses challenges to the complete coverage of the field in the dental curriculum [24]. While endless amounts of medical facts are unlikely to significantly improve the general care of the dental patient, the challenge is to adequately prepare students to manage patients with medical problems within the teaching time available. Focus of internal medicine teaching of both dental students and graduates on the most commonly encountered medical conditions aids the education process [22]. Demonstration of relevance of internal medicine teaching and importance of relevant case-based instruction has been emphasized in the literature [25]. This approach is reliant on the understanding of patient comorbidities the dental students are likely to encounter.

To date, little is known about medical comorbidities and medication usage of Australian dental patients. In this study we presented a uniquely detailed analysis of the medical status of patients seen by the dental students in Western Australia. We reported a high prevalence of systemic diseases in our study population, which can be attributed to the age of the participants and their association with a public institution. This is in keeping with other studies [10,12,13], which have also shown that older patients and those attending public dental facilities exhibit increased prevalence of systemic disease compared to their younger counterparts or those seeking treatment in the private sector. Eligibility at the OHCWA is defined by lower socioeconomic status, which in turn is linked to poorer health [26].

Cardiovascular disease (37.9\%), allergy (32.3\%), mental health disorders (29.4\%) musculoskeletal (23.0\%) endocrine $(22.4 \%)$ and respiratory disease $(17.8 \%)$ constituted the most common conditions reported with the pattern of medication usage paralleling the diseases reported. This is in keeping with other studies [1-16] and also reflects the most common chronic diseases in Australia [27]. Each of those conditions and associated medication use can not only contribute to the presentation of numerous intraoral pathosis but also affect the safe delivery of dental treatment. While dental treatment may potentially exacerbate the underlying medical condition; underlying medical conditions may also 
Table 3 The effect of age group, gender, smoking and alcohol use on positive medical history and medication use

\begin{tabular}{|c|c|c|c|}
\hline Variable & $\begin{array}{l}\text { Male } \\
\mathrm{N}=394 \text { (\%) }\end{array}$ & $\begin{array}{l}\text { Female } \\
\mathrm{N}=479 \text { (\%) }\end{array}$ & $\begin{array}{l}p \text { value (Chi- } \\
\text { square test) }\end{array}$ \\
\hline \multicolumn{4}{|c|}{ Medical history } \\
\hline \multicolumn{4}{|c|}{ Age group (years) } \\
\hline 25-34 & $34 / 40(85.0)$ & $39 / 65(60.0)$ & 0.0069 \\
\hline $35-44$ & 41/54 (75.9) & 60/77 (77.9) & 0.7890 \\
\hline $45-54$ & $51 / 61(83.6)$ & 63/76 (82.9) & 0.9118 \\
\hline $55-64$ & 60/66 (90.9) & $70 / 81(86.4)$ & 0.3972 \\
\hline $65-74$ & 106/112 (94.6) & $108 / 116(93.1)$ & 0.6284 \\
\hline $75-84$ & $50 / 52(96.2)$ & $51 / 51(100.0)$ & $0.4951^{*}$ \\
\hline $85+$ & 8/9 (88.9) & $12 / 13(92.3)$ & $1.0^{*}$ \\
\hline \multicolumn{4}{|l|}{ Medication } \\
\hline \multicolumn{4}{|c|}{ Age group (years) } \\
\hline $25-34$ & $27 / 40(67.5)$ & 36/65 (55.4) & 0.2185 \\
\hline $35-44$ & $35 / 54(64.8)$ & $52 / 77(67.5)$ & 0.7458 \\
\hline $45-54$ & 43/61 (70.5) & $58 / 76(76.3)$ & 0.4415 \\
\hline $55-64$ & $53 / 66(80.3)$ & 68/81 (84.0) & 0.5643 \\
\hline $65-74$ & 101/112 (90.2) & $107 / 115(93.0)$ & 0.4358 \\
\hline $75-84$ & $50 / 52(96.2)$ & $50 / 51(98.0)$ & $1.0^{*}$ \\
\hline $85+$ & 8/9 (88.9) & $11 / 13(84.6)$ & $1.0^{*}$ \\
\hline \multicolumn{4}{|c|}{ Medical history } \\
\hline \multicolumn{4}{|c|}{ Tobacco use } \\
\hline Never & $147 / 168(87.5)$ & 259/313 (82.8) & 0.1708 \\
\hline Current & $99 / 109$ (90.8) & 74/92 (80.4) & 0.0340 \\
\hline Ex-user & 104/117 (88.9) & 69/73 (94.5) & 0.1859 \\
\hline \multicolumn{4}{|l|}{ Medication } \\
\hline \multicolumn{4}{|c|}{ Tobacco use } \\
\hline Never & 130/168 (77.4) & $244 / 313(78.2)$ & 0.8355 \\
\hline Current & $87 / 109$ (79.8) & 75/92 (81.5) & 0.7607 \\
\hline Ex-user & $100 / 117(85.5)$ & 62/73 (84.9) & 0.9189 \\
\hline \multicolumn{4}{|c|}{ Medical history } \\
\hline \multicolumn{4}{|c|}{ Alcohol use } \\
\hline Never & 158/177 (89.3) & $230 / 280(82.1)$ & 0.0383 \\
\hline Current & 181/203 (89.2) & $164 / 190(86.3)$ & 0.3891 \\
\hline Ex-user & $11 / 14(78.6)$ & $8 / 8(100.0)$ & $0.2727^{*}$ \\
\hline \multicolumn{4}{|l|}{ Medication } \\
\hline \multicolumn{4}{|c|}{ Alcohol use } \\
\hline Never & 145/177 (81.9) & $222(79.6)$ & 0.5370 \\
\hline Current & $161 / 203(79.3)$ & $151 / 190(79.5)$ & 0.9681 \\
\hline Ex-user & $11 / 14(78.6)$ & $8 / 8(100.0)$ & $0.2727^{*}$ \\
\hline
\end{tabular}

*Fisher's Exact test

increase the risk of dental treatment complications such as bleeding or infection. Gender differences were observed only with respect to allergy and mental health disorders, with women more commonly affected. This is consistent with Australian data, where women experience a higher prevalence of mental health disorders
Table 4 The effect of IRSAD ${ }^{a}$ (mean, standard deviation, number) on positive medical history and medication use

\begin{tabular}{llll}
\hline Variable & Male $(\mathbf{N}=\mathbf{3 9 4})$ & Female $(\mathbf{N}=479)$ & $\begin{array}{l}\boldsymbol{p} \text { value (Chi- } \\
\text { square test) }\end{array}$ \\
\hline \multicolumn{2}{l}{$\begin{array}{l}\text { Medical history } \\
\text { No }\end{array}$} & & \\
Yes & $1035[60.1], 44$ & $1025[64.7], 76$ & 0.4117 \\
Medication & $1038[64.3], 350$ & $1031[67.3], 403$ & 0.1335 \\
No & $1029[63.7], 77$ & $1034[67.6], 96$ & 0.6230 \\
Yes & $1040[63.7], 317$ & $1029[66.8], 382$ & 0.0298
\end{tabular}

a IRSAD-The Index of Relative Socio-Economic Advantage and Disadvantage (Australian Bureau of Statistics)

then men [28]. Women were also twice as likely to take antidepressant medication.

Medication use was reported by $80 \%$ of study participants. This is significant given the potential for dental treatment complications and drug interactions. Of note, $17 \%$ of study participants reported taking anticoagulants, with men significantly more likely to be affected ( $p=0.0033$; odds ratio [95\% CI] 0.59 [0.41$0.84])$. Although this figure is significantly higher than is reported in the literature $[6,7]$, it is not unexpected give the high prevalence of cardiovascular and nervous system disease in the population studied. Our study participants were also specifically questioned about bleeding during medical history taking and were therefore potentially more likely to recall taking such medication. Drug interactions are an important consideration in dentistry [29-31]. Interactions between adrenaline used in dental local anaesthetics and medications such as tricyclic antidepressants and betablockers are well recognized [29]. In our study 1 in 10 participants reported the use of such medication. Antimicrobials used in dentistry such as erythromycin, clarithromycin, azolic antifungal agents and metronidazole affect the cytochrome P450 system by interacting with various substrates including CYP3A4, CYP2C9 and CYP1A2 [30]. This leads to potentially serious interactions with numerous medications including antihistamines, anticoagulants, antiepileptics, antidepressants, antipsychotics, anticonvulsants, antiviral agents, drugs for dyslipidaemia, calcium channel blockers, immunosuppressants, opiates and benzodiazepines [30]. Many of these were commonly taken by our study population (Table 2). Analgesic agents including paracetamol, selective and non-selective non-steroidal anti-inflammatory drugs (NSAIDs) and narcotics are often prescribed in the dental setting. Opiates exhibit additive sedative and respiratory depressor effects with other central nervous system depressors [31]. They also react with selective serotonin reuptake inhibitors and 
serotoninergic medication [31]. Given that in our study $23 \%$ of study participants reported using antidepressant medications, these are important interactions for dentist to be aware of. Prescription of NSAIDs in hypertensive patients treated with angiotensin-converting enzyme (ACE) inhibitors, beta-blockers, diuretics and angiotensin receptor antagonists can increase bleeding after surgery [31]. One in 10 of our study participants reported using ACE inhibitors and 1 in 10 beta-blockers. The interaction between paracetamol and warfarin [31] is also important to remember in view of the prevalent use of anticoagulants by dental patients.

Aside from treatment complications and drug interactions, medication usage is additionally associated with numerous adverse effects on oral health [32]. These include (but are not limited to): xerostomia and salivary gland hypofunction, dysgeusia, angioedema, mucosal discolouration, gingival hyperplasia, ulceration, erythema multiforme and lichenoid drug reactions [32]. Majority of the medications taken by our study participants have been linked to some type of an adverse oral health affect [32]. Given the prevalence of medication usage among dental patients, the importance of adequate teaching of pharmacology to the dental students cannot be overstated.

Teaching internal medicine and its relevance to the dental setting requires in-depth knowledge of both disciplines and can be challenging. Interdisciplinary exposure is therefore emphasized [25]. Engagement of medical and dental clinicians in the teaching of the most common medical problems encountered in the dental setting can facilitate the most efficient use of time and resources and will broaden the students' educational experience.

Our study was based on examination of patient records generated from information supplied by the participants and is therefore limited by the accuracy of data recorded. Consideration should also be given to a potential selection bias. Although we have minimized the study burden on our participants by employing an opt-out option from participation, it is possible that the healthier individuals were more likely to make the effort to opt-out from the study. Finally, our study is limited to the fact it was based on a population derived from a tertiary referral centre providing subsidized dental services to the members of the public who meet eligibility criteria governed by their socioeconomic status. Results of this study should therefore be considered in the setting of the lower socioeconomic status of the study participants compared to the general Western Australian population. While the population presented in our study is more likely to present with medical conditions and report medication use compared to the wider population that a general dentist is likely to encounter in a private practice setting; it does illustrate the type of medical problems the dental students and dentists are likely to encounter and must be able to manage. It highlights areas on which the internal medicine teaching should focus, to ensure that dental students are well placed to confidently manage patients upon graduation. Finally, this study brings to light the broad experience opportunities that the dental students encounter and the teaching opportunities that exist in a Western Australian tertiary referral centre. Identification of the commonly encountered medical conditions by the dental students has the potential to lay foundation for an integrated case-based program of internal medicine which enhances students' interest and study motivation through demonstration of relevance.

\section{Conclusion}

This study presented a uniquely detailed analysis of the medical status of individuals presenting for dental treatment to a Western Australian tertiary referral centre. Eighty six percent of the participants reported having a medical condition with $80 \%$ taking at least one medication. Cardiovascular disease, allergy, mental health disorders, musculoskeletal, endocrine and respiratory disease constituted the most common conditions reported, highlighting the importance of the clinicians' knowledge of internal medicine and its application to the dental setting.

\section{Abbreviations}

ACE: Angiotensin-converting enzyme; IRSAD: Index of Relative Socio-economic Advantage and Disadvantage; NSAID: Non-steroidal anti-inflammatory drugs; OHCWA: Oral Health Centre of Western Australia; STROBE: The Strengthening the Reporting of Observational Studies in Epidemiology.

\section{Acknowledgements}

We would like to thank all our patients for their willing participation as well as the University of Western Australia Dental School and the Australian Dental Research Foundation for funding.

\section{Authors' contributions}

AMF and OK were responsible for the study concept and design; data acquisition and preparation of the manuscript. RP provided all the statistical analysis and contributed to the manuscript. All authors read and approved the final manuscript.

\section{Funding}

This study was funded by the University of Western Australia Dental School and the Australian Dental Research Foundation. Funding covered the costs of generating project documents, postage and all the statistical analysis.

\section{Availability of data and materials}

The data that support the findings of this study are available on reasonable request from the corresponding author. The data are not publicly available due to privacy or ethical restrictions.

\section{Ethics approval and consent to participate}

This study was approved by the Human Research Ethics Committee of the University of Western Australia (RA/4/20/4435). Participation in the study was voluntary. All subjects were mailed documents about the research project with the option of opting out to participate, as per the approved protocol by 
the Human Research Ethics Committee of the University of Western Australia. All subjects opting out from participation did so in writing.

\section{Consent for publication}

Not applicable.

\section{Competing interests}

All authors reported no conflicts of interest.

\section{Author details}

1 UWA Dental School, The University of Western Australia, 17 Monash Avenue, Nedlands, WA 6009, Australia. ${ }^{2}$ School of Occupational Therapy, Social Work and Speech Pathology, Curtin University, Bentley, WA 6102, Australia.

\section{Received: 8 July 2020 Accepted: 15 October 2020}

Published online: 21 October 2020

\section{References}

1. Fenlon MR, McCartan BE. Medical status of patients attending a primary care dental practice in Ireland. J Ir Dent Assoc. 1991;37(3-4):75-7.

2. Al-Bayaty HF, Murti PR, Naidu RS, Matthews R, Simeon D. Medical problems among dental patients at the school of dentistry, the university of the West Indies. J Dent Educ. 2009;73(12):1408-14.

3. Humbert A, Schmage P, Harendza S. Internal diseases encountered by dental students while treating dental patients during undergraduate training. BMC Med Educ. 2018;18(1):149.

4. de Jong KJ, Oosting J, Abraham-Inpijn L. Medical risk classification of dental patients in The Netherlands. J Public Health Dent. 1993;53(4):219-22.

5. Khader YS, Alsaeed O, Burgan SZ, Amarin ZO. Prevalence of medical conditions among patients attending dental teaching clinics in northern Jordan. J Contemp Dent Pract. 2007;8(1):60-7.

6. Radfar L, Suresh L. Medical profile of a dental school patient population. J Dent Educ. 2007;71(5):682-6.

7. Cottone JA, Kafrawy AH. Medications and health histories: a survey of 4,365 dental patients. J Am Dent Assoc. 1979;98(5):713-8.

8. Eggleston DJ. The value of a simple medical questionnaire in dentistry. Aust Dent J. 1977;22(3):160-4.

9. Peacock ME, Carson RE. Frequency of self-reported medical conditions in periodontal patients. J Periodontol. 1995;66(11):1004-7.

10. Georgiou TO, Marshall RI, Bartold PM. Prevalence of systemic diseases in Brisbane general and periodontal practice patients. Aust Dent J. 2004;49(4):177-84.

11. Rhodus NL. The frequency and profile of medically compromising conditions in an dental school patient population. Gen Dent. 1988;36(3):219-22.

12. Nery EB, Meister F Jr, Ellinger RF, Eslami A, McNamara TJ. Prevalence of medical problems in periodontal patients obtained from three different populations. J Periodontol. 1987;58(8):564-8.

13. Jainkittivong A, Aneksuk V, Langlais RP. Medical health and medication use in elderly dental patients. J Contemp Dent Pract. 2004;5(1):31-41.

14. Almas K, Awartani FA. Prevalence of medically compromised patients referred for periodontal treatment to a teaching hospital in Central Saudi Arabia. Saudi Med J. 2003;24(11):1242-5.

15. Fitzgerald J, Epstein JB, Donaldson M, Schwartz G, Jones C, Fung K. Outpatient medication use and implications for dental care: guidance for contemporary dental practice. J Can Dent Assoc. 2015;81:f10.
16. Bhateja S. High prevalence of cardiovascular diseases among other medically compromised conditions in dental patients: a retrospective study. J Cardiovasc Dis Res. 2012;3(2):113-6.

17. Miller C. AAOM Clinical Practice Statement: Subject: Medical History. Oral Surg Oral Med Oral Pathol Oral Radiol. 2016;121(6):618-9.

18. Staff TRM. Health History Vital in Assessing Patients, Reducing Risk. J Calif Dent Assoc 2015; 43(7):395-396, 398.

19. Hadden AM, and the FCE, Record-Keeping Working G: Clinical examination \& record-keeping: Part 2: history taking. Br Dent J 2017; 223(11):823-825

20. Miyatake $Y$, Kazama M, Isoda M, Nejima J. Internal medicine education in dentistry: knowledge required varies according to dental specialty. Eur J Dent Educ. 2004;8(1):18-23.

21. Carvalho RM, Costa LR, Marcelo VC. Brazilian dental students' perceptions about medical emergencies: a qualitative exploratory study. J Dent Educ. 2008;72(11):1343-9.

22. Dornan T, Bundy C. What can experience add to early medical education? Consensus survey. BMJ. 2004;329(7470):834.

23. von Elm E, Altman DG, Egger M, Pocock SJ, Gotzsche PC, Vandenbroucke $J$, Initiative S. Strengthening the Reporting of Observational Studies in Epidemiology (STROBE) statement: guidelines for reporting observational studies. BMJ. 2007;335(7624):806-8.

24. Tack CJ, Plasschaert AJ. Student evaluation of a problem-oriented module of clinical medicine within a revised dental curriculum. Eur J Dent Educ. 2006;10(2):96-102.

25. Dennis MJ, Bennett JD, DeLuke DM, Evans EW, Hudson JW, Nattestad A, Ness GM, Yeung A. Improving the Medical Curriculum in Predoctoral Dental Education: Recommendations From the American Association of Oral and Maxillofacial Surgeons Committee on Predoctoral Education and Training. J Oral Maxillofac Surg. 2017;75(2):240-4.

26. Schofield DJ, Callander EJ, Shrestha RN, Passey ME, Kelly SJ, Percival R. Multiple chronic health conditions and their link with wealth assets. Eur J Public Health. 2015;25(2):285-9.

27. AlHW: Australian Institute of Health and Welfare. Australian Burden of Disease Study: impact and causes of illness and death in Australia 2015 Summary report. Australian Burden of Disease Study series no. 18.Cat. no. BOD 21. Canberra: AlHW. 2019.

28. AlHW: Australian Institute of Health and Welfare. Mental health servicesin brief 2019. Cat. no. HSE 228. Canberra: AlHW. 2019.

29. Gomez-Moreno G, Guardia J, Cutando A, Calvo-Guirado JL. Pharmacological interactions of vasoconstrictors. Med Oral Patol Oral Cir Bucal. 2009a;14(1):E20-27.

30. Gomez-Moreno G, Guardia J, Cutando A, Calvo-Guirado JL. Pharmacological interactions of anti-microbial agents in odontology. Med Oral Patol Oral Cir Bucal. 2009b;14(3):E123-128.

31. Gomez-Moreno G, Guardia J, Cutando A, Calvo-Guirado JL. Pharmacological interactions of anti-inflammatory-analgesics in odontology. Med Oral Patol Oral Cir Bucal. 2009c;14(2):E81-89.

32. Ciancio SG. Medications' impact on oral health. J Am Dent Assoc 2004; 135(10):1440-1448 (quiz 1468-1449).

\section{Publisher's Note}

Springer Nature remains neutral with regard to jurisdictional claims in published maps and institutional affiliations. 